\title{
The solar photospheric abundance of hafnium and thorium
}

\section{Results from $\mathrm{CO}^{5} \mathrm{BOLD}$ 3D hydrodynamic model atmospheres}

\author{
E. Caffau ${ }^{1}$, L. Sbordone ${ }^{2,1}$, H.-G. Ludwig ${ }^{2,1}$, P. Bonifacio ${ }^{2,1,3}$, M. Steffen ${ }^{4}$, and N. T. Behara ${ }^{2,1}$ \\ 1 GEPI, Observatoire de Paris, CNRS, Université Paris Diderot, Place Jules Janssen, 92190 Meudon, France \\ e-mail: Elisabetta.Caffau@obspm.fr \\ 2 CIFIST Marie Curie Excellence Team \\ 3 Istituto Nazionale di Astrofisica, Osservatorio Astronomico di Trieste, via Tiepolo 11, 34143 Trieste, Italy \\ 4 Astrophysikalisches Institut Potsdam, An der Sternwarte 16, 14482 Potsdam, Germany
}

Received 21 February 2008 / Accepted 17 March 2008

\section{ABSTRACT}

\begin{abstract}
Context. The stable element hafnium (Hf) and the radioactive element thorium (Th) were recently suggested as a suitable pair for radioactive dating of stars. The applicability of this elemental pair needs to be established for stellar spectroscopy.

Aims. We aim at a spectroscopic determination of the abundance of $\mathrm{Hf}$ and $\mathrm{Th}$ in the solar photosphere based on a $\mathrm{CO} \mathrm{BOLD}^{5}$ 3D hydrodynamical model atmosphere. We put this into a wider context by investigating 3D abundance corrections for a set of Gand F-type dwarfs.

Methods. High-resolution, high signal-to-noise solar spectra were compared to line synthesis calculations performed on a solar $\mathrm{CO}^{5} \mathrm{BOLD}$ model. For the other atmospheres, we compared synthetic spectra of $\mathrm{CO}^{5} \mathrm{BOLD} 3 \mathrm{D}$ and associated $1 \mathrm{D}$ models.

Results. For Hf we find a photospheric abundance $A(\mathrm{Hf})=0.87 \pm 0.04$, in good agreement with a previous analysis, based on 1D model atmospheres. The weak Th II $401.9 \mathrm{~nm}$ line constitutes the only Th abundance indicator available in the solar spectrum. It lies in the red wing of a Ni-Fe blend exhibiting a non-negligible convective asymmetry. Accounting for the asymmetry-related additional absorption, we obtain $A(\mathrm{Th})=0.08 \pm 0.03$, consistent with the meteoritic abundance, and about 0.1 dex lower than obtained in previous photospheric abundance determinations.

Conclusions. Only for the second time, to our knowledge, has a non-negligible effect of convective line asymmetries on an abundance derivation been highlighted. Three-dimensional hydrodynamical simulations should be employed to measure Th abundances in dwarfs if similar blending is present, as in the solar case. In contrast, 3D effects on Hf abundances are small in G- to mid F-type dwarfs and sub-giants, and 1D model atmospheres can be conveniently used.
\end{abstract}

Key words. Sun: abundances - stars: abundances - hydrodynamics

\section{Introduction}

The determination of the ages of the older stellar populations in the Galaxy is fundamental for constraining its buildup mechanisms and determining the associated timescales. A variety of dating techniques have been developed, the majority relying on comparing the colour-magnitude-diagram of a stellar population with theoretical isochrones or "fiducial lines" derived from other observed populations (typically globular clusters).

Long lived unstable isotopes of heavy elements such as ${ }^{238} \mathrm{U}$ and ${ }^{232} \mathrm{Th}$ provide an independent method of determining stellar ages. Such elements are generally produced through neutron capture (specifically rapid neutron capture or r-process) and then decay. Their abundance at the present time thus provides a direct estimate of the time passed since they were produced, if their initial abundance can somehow be inferred. This is typically accomplished by measuring some other stable element with a common nucleosynthetic origin together with the radioactive element. Europium (Eu) has long been a typical choice, since its abundance relative to the abundance of thorium can be established from r-process enrichment models (see François et al. 1993; Cowan et al. 1999; Ivans et al. 2006). Sneden et al. (2000) used the $[\mathrm{Th} / \mathrm{Eu}]$ abundance ratio to estimate the age of the old, metal-poor globular cluster M 15 to $14 \pm 3$ Gyr. The study was made easier by the fact that M 15 shows strong r-process element enhancement $([\mathrm{Eu} / \mathrm{Fe}]=1.15,[\mathrm{Th} / \mathrm{Fe}]=0.81$, Sneden et al. 2000). Nevertheless, Eu presents some drawbacks as a cosmochronometric reference element: while easily measurable, its atomic mass is substantially different from the one of Th and $U$. This generally increases uncertainties. In fact, decaying and stable reference elements should both be formed in the same event, which - for heavy n-capture species - can be safely assumed only if the atomic masses of the two elements are similar. This means in practise that, when a stable reference element is used, it should be as massive as possible. Alternatively, where $U$ is measured, the U/Th ratio can be used since the two have very different half-life times. The measurement of $U$ is nevertheless extremely difficult and has been possible only in two metal-poor r-enhanced stars (Cayrel et al. 2001; Hill et al. 2002; Frebel et al. 2007).

Recently, hafnium (Hf, $Z=72$ ) has been suggested as a suitable stable reference element. The Hf production rate via the r-process is believed to be strictly tied to the one of thorium, so that their abundance ratio is almost constant regardless of the neutron density in the generating Super Novae (SN, Kratz et al. 2007). Hafnium is more massive than $\mathrm{Eu}$ (stable Eu isotopes are 151 and 153 , while $A_{\mathrm{Hf}} \approx 178$ ) and can be measured from a set of lines in the blue part of the visual spectrum (the most suitable 
ones being at 391.8 and $409.3 \mathrm{~nm}$ ) for which accurate transition data have been recently measured (Lawler et al. 2007). On the down-side, Hf is believed to be efficiently produced by s-process (about 55\% of the meteoritic Hf content in the solar system is believed to have been produced by the s-process, see Arlandini et al. 1999). As a consequence, it can be effectively employed as a reference only where s-process enrichment can be considered negligible, or if the s-process contribution can be reliably modelled.

Although in principle promising, Hf/Th dating is not free of drawbacks. In the first place an uncertainty of $0.1 \mathrm{dex}$ in [Th/Hf] implies about $4.7 \mathrm{Gyr}$ (see also Ludwig et al. 2008a) uncertainty in the dating. Second, the theoretical prediction of strong ties between Th and Hf production needs to be verified by observations. Third, the applicability of the method to normal (i.e. not r-process enhanced) stars needs some testing: especially Th lines become relatively weak and significant blends can be prejudicial especially in more metal-rich objects.

With the prospect of using hafnium and thorium for dating purposes, we decided to determine the solar hafnium and thorium abundances using hydrodynamical model atmospheres. We anticipate that $3 \mathrm{D}$ effects are found to be non-negligible for $\mathrm{Th}$, and mainly due to the convection-induced asymmetry of blending lines.

In the solar spectrum, the measurable lines of Hf are from the singly ionised atom, and are often blended with other photospheric absorption lines. As a consequence, solar photospheric abundance determinations are scarce in the literature. Russell (1929) determined the photospheric hafnium solar abundance to be 0.90 . Recently, Lawler et al. (2007) measured Hf lifetimes for several lines, four of which were used to determine a solar photospheric hafnium abundance of $0.88 \pm 0.08$. Andersen et al. (1976) considered nine lines of Hf II (two of which are in common with Lawler et al. 2007) and obtained from a Kitt Peak National Observatory centre-disc spectrum a solar photospheric hafnium abundance of $0.88 \pm 0.08$. Values of the meteoritic hafnium abundance cited in literature include: $0.73 \pm 0.01$, Anders \& Grevesse (1989); $0.75 \pm 0.02$ Grevesse \& Sauval (1998); and $0.77 \pm 0.04$ Lodders (2003).

Thorium is measurable in the Sun from one single line of Th II at $401.9 \mathrm{~nm}$, which is heavily blended. Solar thorium abundances in literature are very rare. Holweger (1980) derived a Th abundance of 0.16, Anders \& Grevesse (1989) report abundances of $0.12 \pm 0.06$ from the $401.9 \mathrm{~nm}$ line due to an unpublished measurement of Grevesse. Lodders (2003) recommends the meteoritic value $A(\mathrm{Th})=0.09 \pm 0.04$ from Grevesse et al. (1996). The meteoritic value is $0.08 \pm 0.02$ according to Anders \& Grevesse (1989). Rocholl \& Jochum (1993) measured $0.0329 \mathrm{Th}$ atoms to $10^{6} \mathrm{Si}$ atoms in carbonaceous chondrites.

\section{Atomic data}

The same four Hf II lines with the same $\log g f$ as Lawler et al. (2007) have been considered (see Table 1). Line parameters for the $401.9 \mathrm{~nm}$ Th II and blending lines in this range are from del Peloso et al. (2005a).

\section{Models}

Our analysis is based on a 3D model atmosphere computed with the $\mathrm{CO}^{5}$ BOLD code (Freytag et al. 2002; Wedemeyer et al. 2004). In addition to the CO ${ }^{5}$ BOLD hydrodynamical simulation we use several 1D models. More details about the
Table 1. Hf II lines considered in this work.

\begin{tabular}{rcrr}
\hline $\begin{array}{r}\text { Wavelength } \\
\mathrm{nm}\end{array}$ & Transition & $\begin{array}{r}E_{\text {low }} \\
\mathrm{eV}\end{array}$ & $\log g f$ \\
\hline 338.983 & $\mathrm{a}^{4} \mathrm{~F}-\mathrm{z}^{2} \mathrm{P}^{0}$ & 0.45 & -0.78 \\
356.166 & $\mathrm{a}^{2} \mathrm{D}-\mathrm{z}^{4} \mathrm{~F}^{0}$ & 0.00 & -0.87 \\
391.809 & $\mathrm{a}^{4} \mathrm{~F}-\mathrm{z}^{4} \mathrm{D}^{0}$ & 0.45 & -1.14 \\
409.315 & $\mathrm{a}^{4} \mathrm{~F}-\mathrm{z}^{4} \mathrm{P}^{0}$ & 0.45 & -1.15 \\
\hline
\end{tabular}

applied models can be found in Caffau et al. (2007a) and Caffau \& Ludwig (2007). As in previous works we call $\langle 3 \mathrm{D}\rangle$ the $1 \mathrm{D}$ model derived by a horizontal and temporal averaging of the 3D CO${ }^{5} \mathrm{BOLD}$ model. The reference $1 \mathrm{D}$ model for the computation of the 3D abundance corrections is a hydrostatic 1D model atmosphere computed with the LHD code (see Caffau

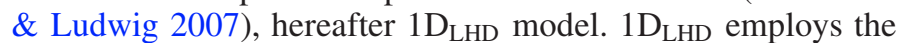
same micro-physics (opacities, equation-of-state, radiative transfer scheme) as $\mathrm{CO}^{5} \mathrm{BOLD}$, and treats the convective energy transport with mixing-length theory. Here, we set the mixinglength parameter to 1.5. We also use the solar ATLAS 9 model computed by Fiorella Castelli ${ }^{1}$ and the Holweger-Müller solar model (Holweger 1967; Holweger \& Müller 1974). The spectrum synthesis code we employ is Linfor $3 \mathrm{D}^{2}$ for all models.

The 3D $\mathrm{CO}^{5} \mathrm{BOLD}$ solar model covers a time interval of $4300 \mathrm{~s}$, represented by 19 snapshots. This time interval should be compared to a characteristic time scale related to convection. Here we choose the sound crossing time over one pressure scale height at the surface $t_{\mathrm{c}}=H_{\mathrm{P}} / c\left(H_{\mathrm{P}}\right.$ : pressure scale height at $\tau_{\text {ross }}=1, c$ : sound speed) which amounts to $17.8 \mathrm{~s}$ in the solar case. Note, that this time scale is not the time over which the convective pattern changes its appearance significantly. This time scale is significantly longer than $t_{\mathrm{c}}$; e.g., Wedemeyer et al. (2004) obtained from simulations an autocorrelation life time of about $120 \mathrm{~s}$ for the convection pattern. However, while $t_{\mathrm{c}}$ does not measure the life time of the convective pattern as such, it provides a reasonably accurate scaling of its life time with atmospheric parameters (e.g., Svensson \& Ludwig 2005). Hence, it can be used for the inter-comparison of different 3D models.

The particular solar model used in this paper has a higher wavelength resolution (12 opacity bins) with respect to the model we used to study the solar phosphorus abundance (Caffau et al. 2007b). The basic characteristics of the 3D models we consider in this work can be found in Table 2.

As in our study of sulphur (see Caffau \& Ludwig 2007) and phosphorus (see Caffau et al. 2007b), we define as "3D correction" the difference in the abundance derived from the $3 \mathrm{D} \mathrm{CO}^{5} \mathrm{BOLD}$ model and the $1 \mathrm{D}_{\mathrm{LHD}}$ model, both synthesised with Linfor3D. We consider also the difference in the abundance derived from the $3 \mathrm{D} \mathrm{CO}^{5} \mathrm{BOLD}$ model and from the $\langle 3 \mathrm{D}\rangle$ model. Since the $3 \mathrm{D} \mathrm{CO}^{5} \mathrm{BOLD}$ and $\langle 3 \mathrm{D}\rangle$ models have, by construction, the same mean temperature structure, this allows us to single out the effects due to the horizontal temperature fluctuations.

\section{Observational data}

The observational data we use consist of two high-resolution, high signal-to-noise ratio spectra of centre disc solar intensity: that of Neckel \& Labs (1984, hereafter referred to as the "Neckel

\footnotetext{
1 http://www. user.oats.inaf.it/castelli/sun/ ap00t5777g44377k1asp.dat

2 http://www .aip.de/ mst/Linfor3D/linfor_3D_manual. $\operatorname{pdf}$
} 
Table 2. The $\mathrm{CO}^{5} \mathrm{BOLD}$ models considered in this work.

\begin{tabular}{rrrrrr}
\hline \hline $\begin{array}{r}T_{\text {eff }} \\
(\mathrm{K})\end{array}$ & $\begin{array}{r}\log g \\
\left(\mathrm{~cm} \mathrm{~s}^{-2}\right)\end{array}$ & {$[\mathrm{M} / \mathrm{H}]$} & $N_{\mathrm{t}}$ & $\begin{array}{r}\text { time } \\
(\mathrm{s})\end{array}$ & $\begin{array}{r}t_{\mathrm{c}} \\
(\mathrm{s})\end{array}$ \\
$(1)$ & $(2)$ & $(3)$ & $(4)$ & $(5)$ & $(6)$ \\
\hline 5770 & 4.44 & 0.0 & 19 & 4300 & 17.8 \\
4980 & 4.50 & 0.0 & 20 & 30800 & 14.8 \\
5060 & 4.50 & -1.0 & 19 & 65600 & 15.1 \\
5430 & 3.50 & 0.0 & 18 & 144000 & 151.0 \\
5480 & 3.50 & -1.0 & 19 & 106800 & 149.3 \\
5930 & 4.00 & 0.0 & 18 & 26400 & 49.8 \\
5850 & 4.00 & -1.0 & 18 & 36600 & 48.9 \\
5870 & 4.50 & 0.0 & 19 & 16900 & 16.0 \\
5920 & 4.50 & -1.0 & 8 & 7000 & 15.7 \\
6230 & 4.50 & 0.0 & 20 & 35400 & 16.3 \\
6240 & 4.50 & -1.0 & 20 & 25000 & 16.1 \\
6460 & 4.50 & 0.0 & 20 & 17400 & 16.4 \\
6460 & 4.50 & -1.0 & 20 & 36200 & 16.3 \\
\hline
\end{tabular}

Note: Cols. (1)-(3) state the atmospheric parameters of the models; Col. (4) the number of snapshots $N_{\mathrm{t}}$ considered; Col. (5) the time interval covered by the selected snapshots; Col. (6) a characteristic timescale $t_{\mathrm{c}}$ of the evolution of the granular flow.

intensity spectrum") and that of Delbouille et al. (1973, hereafter referred to as the "Delbouille intensity spectrum"3). We further use the disc-integrated solar flux spectrum of Neckel \& Labs (1984) and the solar flux spectrum of Kurucz $(2005)^{4}$, referred to as the "Kurucz flux spectrum".

\section{Data analysis}

\subsection{Hafnium}

The computation of a $3 \mathrm{D}$ synthetic spectrum with Linfor3D is rather time consuming and the spectral-synthesis code can handle easily only a few tens of lines. The $\log g f$ values of the lines blending the Hf lines and lying nearby are not well known, and the synthetic spectra do not reproduce the observed solar spectrum well in that region. To fit the line profiles Lawler et al. (2007) changed the $\log g f$ of some lines blending the Hf II lines, but did not make available the values used. In any case, solar $\log g f$ values derived from $1 \mathrm{D}$ and 3D fitting are not guaranteed to be the same. Our computational power is insufficient at the moment to enable us to fit 3D synthetic spectra to an observed spectrum by changing the $\log g f$ values of various lines. Consequently, we could not produce solar $\log g f$ for the blending lines and fit the line profiles with a 3D model grid. Instead of fitting astrophysical solar $\log g f$ of the blending features, we introduced blending components in the process of measuring $E W$. We thus took advantage of the deblending mode of the IRAF task splot. The corresponding Hf abundance is then derived from the curves of growth computed with Linfor3D for the various lines. The results of Lawler et al. (2007) are used as a check for our measurements. The abundances we obtain from the Holweger-Müller (HM) model are in agreement with their measurement. The results for all spectra and individual lines are presented in Table 3.

We determine the following hafnium abundances: $0.870 \pm$ 0.038 if we consider only the disc-centre intensity spectra, $0.818 \pm 0.005$ according to the solar flux spectra, and $0.856 \pm$ 0.040 if we consider both the intensity and the flux spectra. As

\footnotetext{
${ }^{3}$ http://bass2000.obspm.fr/solar_spect.php

${ }^{4}$ http://kurucz.harvard.edu/sun.html
}
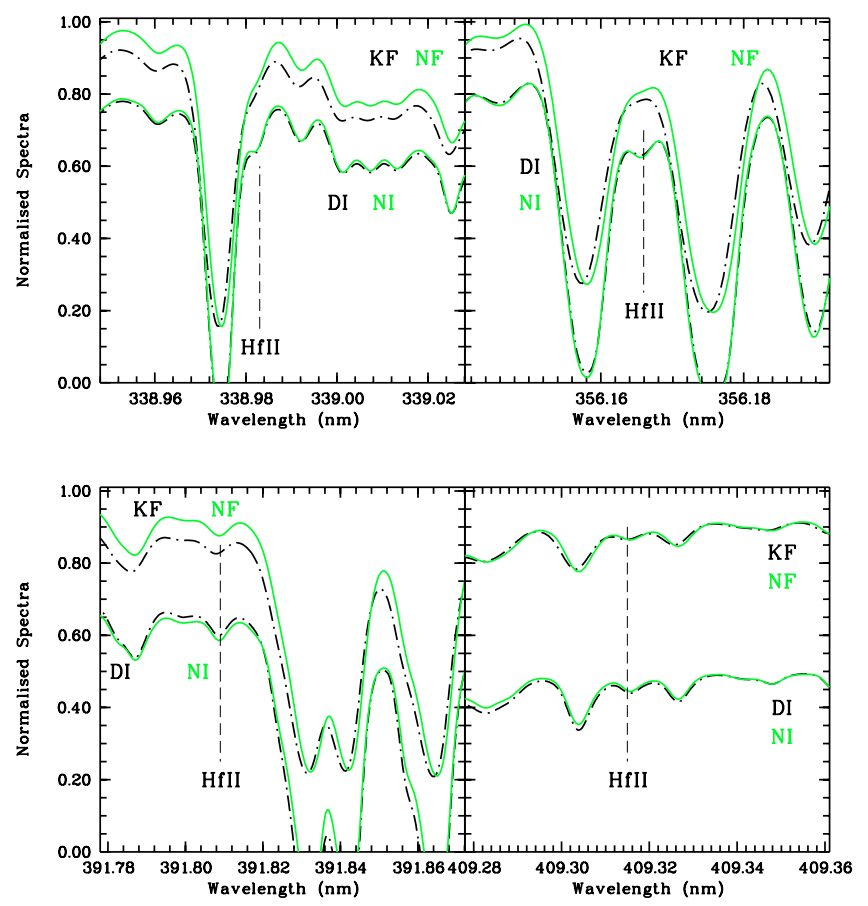

Fig. 1. Four different observed solar spectra, displayed for four different spectral windows, each containing a $\mathrm{Hf}$ II line, indicated by a thin dashed vertical line. For clarity, the intensity spectra, labelled as DI (Delbouille Intensity) and NI (Neckel Intensity), are shifted down by -0.2 units, while the disk-integrated spectra, KF (Kurucz Flux) and NF (Neckel Flux), are shifted up by 0.2 units for the $356.1 \mathrm{~nm}$ and $391.8 \mathrm{~nm}$ lines. Except for the DI spectrum, the original normalisation has been retained. Note that the different spectra do not always agree perfectly.

final uncertainty we took the line to line rms scatter over all spectra. Equivalent widths can be measured in both flux spectra only for the $409.3 \mathrm{~nm}$ hafnium line, which is nearly free of blends. Only the Neckel flux spectrum can be considered for the Hf line at $391.8 \mathrm{~nm}$, due to the fact that the larger broadening of flux spectra with respect to intensities makes the measurement more difficult, if not impossible. Additionally, NLTE corrections may be different in intensity and flux spectra. For these reasons, and to have a consistent comparison with the results of Lawler et al. (2007), we determine the solar hafnium abundance from the average value stemming from the disc-centre spectra. Our results are in good agreement with the results of Lawler et al. (2007); we obtain $A(\mathrm{Hf})=0.878 \pm 0.040$ from the Holweger-Müller model, considering only disc-centre spectra, which is to compare to their value of $0.88 \pm 0.02$.

The 3D abundance corrections for Hf are very small. The highest value, $0.037 \mathrm{dex}$, is the one for the strongest line. As previously mentioned, a 3D correction is the difference in abundance obtained from the $3 \mathrm{D}$ model and the $1 \mathrm{D}_{\mathrm{LHD}}$ model. If one chooses an ATLAS or the Holweger-Müller model as a 1D reference model, the 3D correction would be even smaller or negative, respectively.

The $\langle 3 \mathrm{D}\rangle$ and $1 \mathrm{D}_{\mathrm{LHD}}$ solar model temperature structures are similar. The relative contribution to the $3 \mathrm{D}$ correction due to different average temperature profiles is small. The $3 \mathrm{D}$ correction is larger for stronger lines, quite insensitive to micro-turbulence, of the same order of magnitude for flux and intensity, and slightly higher for intensity. 
Table 3. Solar hafnium abundances from the various observed spectra.

\begin{tabular}{|c|c|c|c|c|c|c|c|c|c|c|c|c|}
\hline$\overline{\text { Spec }}$ & $\begin{array}{l}\text { Wavelength } \\
\text { nm } \\
\text { (2) }\end{array}$ & $\begin{array}{l}E W \\
\text { pm } \\
(3)\end{array}$ & $\begin{array}{c}3 \mathrm{D} \\
(4)\end{array}$ & $\begin{array}{r}\langle 3 \mathrm{D}\rangle \\
(5)\end{array}$ & $\begin{array}{c}A(\mathrm{Hf}) \\
1 \mathrm{D}_{\mathrm{LHD}} \\
(6)\end{array}$ & $\begin{array}{r}1 \mathrm{D}_{\text {ATLAS }} \\
\text { (7) }\end{array}$ & $\begin{array}{r}1 \mathrm{D}_{\mathrm{HM}} \\
(8)\end{array}$ & $\begin{array}{r}\sigma \\
\text { dex } \\
(9)\end{array}$ & $\begin{array}{l}\text { Ref. } \\
\text { (10) }\end{array}$ & $\begin{array}{r}3 \mathrm{D}-1 \mathrm{D}_{\mathrm{LHD}} \\
\mathrm{dex} \\
(11)\end{array}$ & $\begin{array}{r}\sigma_{\langle E W\rangle} \\
\% \\
(12)\end{array}$ & $\begin{array}{r}\sigma_{\mathrm{A}} \\
\operatorname{dex} \\
(13)\end{array}$ \\
\hline NI & 338.9 & 0.489 & 0.911 & 0.895 & 0.883 & 0.900 & 0.914 & 0.02 & & 0.029 & 0.29 & 0.0013 \\
\hline DI & 338.9 & 0.477 & 0.899 & 0.883 & 0.871 & 0.886 & 0.903 & 0.02 & 0.91 & 0.029 & & \\
\hline NI & 356.2 & 0.845 & 0.819 & 0.801 & 0.781 & 0.799 & 0.821 & 0.01 & & 0.037 & 0.31 & 0.0014 \\
\hline DI & 356.2 & 0.851 & 0.822 & 0.805 & 0.785 & 0.803 & 0.824 & 0.02 & 0.85 & 0.037 & & \\
\hline NF & 391.8 & 0.318 & 0.823 & 0.827 & 0.815 & 0.836 & 0.848 & 0.05 & & 0.008 & 0.26 & 0.0011 \\
\hline NI & 391.8 & 0.302 & 0.911 & 0.899 & 0.893 & 0.912 & 0.925 & 0.02 & & 0.018 & 0.27 & 0.0012 \\
\hline DI & 391.8 & 0.274 & 0.867 & 0.854 & 0.849 & 0.868 & 0.881 & 0.05 & 0.91 & 0.018 & & \\
\hline KF & 409.3 & 0.317 & 0.814 & 0.818 & 0.805 & 0.826 & 0.838 & 0.07 & & 0.010 & 0.26 & 0.0011 \\
\hline NF & 409.3 & 0.318 & 0.816 & 0.820 & 0.806 & 0.828 & 0.840 & 0.07 & & 0.010 & & \\
\hline NI & 409.3 & 0.292 & 0.886 & 0.874 & 0.865 & 0.884 & 0.899 & 0.02 & & 0.020 & 0.26 & 0.0011 \\
\hline DI & 409.3 & 0.268 & 0.847 & 0.835 & 0.827 & 0.846 & 0.860 & 0.04 & 0.86 & 0.020 & & \\
\hline
\end{tabular}

Note: Abundances and their uncertainties - if not noted otherwise - are given on the usual logarithmic spectroscopic scale where $A(\mathrm{H})=12$. Column (1) is an identification flag, DI means Delbouille and NI Neckel disc-centre spectrum, NF Neckel and KF Kurucz flux spectrum; Col. (4): hafnium abundance, $A(\mathrm{Hf})$, according to the $\mathrm{CO}^{5} \mathrm{BOLD} 3 \mathrm{D}$ model; Cols. (5)-(8): $A(\mathrm{Hf})$ from 1D models, with $\xi$, micro-turbulence, of $1.0 \mathrm{~km} \mathrm{~s}{ }^{-1}$; Col. (9): uncertainties related to $E W$ measurement; Col. (10) Lawler et al. (2007); Col. (11): 3D corrections; Col. (12): uncertainty of the theoretical equivalent width due to the limited statistics obtained from the 3D model; Col. (13): uncertainty in abundance due to the model uncertainty in equivalent width.

Table 4. Solar thorium abundances derived from the EWs of Lawler et al. (1990).

\begin{tabular}{|c|c|c|c|c|c|c|c|c|c|c|}
\hline \multirow{3}{*}{$\begin{array}{l}\text { spec } \\
\text { (1) }\end{array}$} & \multirow{3}{*}{$\begin{array}{l}E W \\
\text { pm } \\
(2)\end{array}$} & \multicolumn{4}{|c|}{$\overline{\overline{A(\mathrm{Th})}}$} & \multirow[t]{2}{*}{$\bar{\sigma}$} & \multirow[t]{2}{*}{ 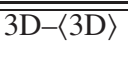 } & \multirow[t]{2}{*}{$3 \mathrm{D}-1 \mathrm{D}_{\mathrm{LHD}}$} & \multirow{3}{*}{$\begin{array}{r}\sigma_{\langle E W\rangle} \\
\% \\
(10)\end{array}$} & \multirow{3}{*}{$\begin{array}{r}\sigma_{\mathrm{A}} \\
\operatorname{dex} \\
(11)\end{array}$} \\
\hline & & 3D & $\langle 3 \mathrm{D}\rangle$ & $1 \mathrm{D}_{\text {LHD }}$ & $1 \mathrm{D}_{\mathrm{HM}}$ & & & & & \\
\hline & & (3) & (4) & (5) & (6) & (7) & (8) & (9) & & \\
\hline Int & 0.415 & 0.168 & 0.164 & 0.148 & 0.190 & 0.022 & 0.004 & 0.0207 & 0.33 & 0.0015 \\
\hline Flux & 505 & 0.135 & 0.151 & 0.130 & 0.172 & & -0.015 & 0.0052 & 0.34 & 0.0015 \\
\hline Int* & 0.355 & 0.095 & 0.092 & 0.075 & 0.118 & 0.026 & 0.003 & 0.0199 & & \\
\hline Flux* & 0.430 & 0.060 & 0.074 & 0.054 & 0.096 & & -0.015 & 0.0060 & & \\
\hline
\end{tabular}

Notes: The contribution to the $E W$ of Co and V, according to the 3D simulation, has been subtracted from the $E W$ s in the last two rows (labelled with *). (1) identification flag to distinguish disc-centre to flux spectrum results; (3) thorium abundance, $A(\mathrm{Th})$, according to the $\mathrm{CO}^{5} \mathrm{BOLD}$ 3D model; (4)-(6) A(Th) from 1D models, with $\xi$, micro-turbulence, of $1.0 \mathrm{~km} \mathrm{~s}^{-1}$; (7) the uncertainty due to the $E W$; (8)-(9) 3D corrections; (10) statistical uncertainty of the theoretical $E W ;(11)$ corresponding uncertainty in abundance.

\subsection{Thorium}

Singly ionised thorium is the dominant ionisation stage in the solar atmosphere, and thorium is measurable in the Sun only through the $401.9 \mathrm{~nm}$ Th II resonance line. Holweger (1980) detected also the $408.6 \mathrm{~nm}$ Th II line, however in our view this line is too weak and blended to be used for quantitative analysis. The $401.9 \mathrm{~nm}$ line happens to fall on the wing of a strong blend of neutral iron and nickel. Additionally, it is blended with a Co I line (about $25 \%$ of the $E W$ of the Th II line) and a weaker V II line (1/5 of the Co line, $1 / 9$ of the Th line in $E W)$. The Co I and V II lines are very close in wavelength to the Th II line, and it is therefore practically impossible to disentangle the blend. Consequently, we do not measure the contribution in $E W$ due to thorium, but instead use the $E W$ s measured by Lawler et al. (1990) to obtain $A(T h)$ (see Table 4, first two lines). However, while Lawler et al. (1990) considered only the contribution of Co I to the blend, we consider also the contribution of V II. To do so we computed from the $\mathrm{CO}^{5} \mathrm{BOLD}$ solar model the $E W$ of the Co I+V II blend, using the atomic data from del Peloso et al. (2005a), and subtracted this value from the $E W$ of Lawler et al. (1990). In this manner, we obtained a smaller solar $A(T h)$ (see last two lines of Table 4). The 3D corrections listed in Table 4 are deduced from the $E W \mathrm{~s}$ alone, and we shall call them in the following "intrinsic" 3D corrections. These are the corrections that would be found if the Th II line were isolated. Since it is instead found in the middle of a rather complex spectral region, other 3D-related effects arise, which are discussed later.
The last two columns of Table 4 provide estimates of the statistical uncertainty of the theoretical $E W$ predicted by the 3D model and the associated uncertainty in the thorium abundance. The 3D model provides only a statistical realisation of the stellar atmosphere, and is thus subject to limited statistics (see Ludwig et al. 2008b). However, as necessary for a reliable derivation of abundances these uncertainties are insignificant and only added for completeness.

Since the ThII line lies on the red wing of the stronger $\mathrm{Fe}$ I+Ni I blend, one can expect that the line asymmetry of the latter contributes in a non-negligible manner to the measured $E W$. Up to now it has generally been considered that convective line asymmetries have no impact on the derived abundances, which is true, if the line is isolated. Cayrel et al. (2007) have demonstrated that such asymmetries are non-negligible for the derivation of the ${ }^{6} \mathrm{Li} /{ }^{7} \mathrm{Li}$ isotopic ratio. From the purely morphological point of view, the case of the Th II resonance line is similar to that of the ${ }^{6} \mathrm{Li}$ I resonance line: a weak line which falls on the red wing of a stronger line, which is asymmetric due to the effects of convection. In order to investigate this effect one has to consider the full line profile and resort to spectrum synthesis.

To gain some insight into the problem we began by comparing synthetic profiles, which included only the Fe I+Ni I blend and the Th II+Co I+V II blend, computed from the CO ${ }^{5}$ BOLD and $1 \mathrm{D}_{\text {LHD }}$ models. In Fig. 2 , the $1 \mathrm{D}_{\mathrm{LHD}}$ flux profile of the $\mathrm{Fe}$ I+Ni I blend has been scaled (since the line strength is different in 1D and 3D) in order to have the same central depth of the $3 \mathrm{D}$ profile. The asymmetry of the red wing is obvious. The 
Table 5. Solar thorium abundance derived from line profile fitting.

\begin{tabular}{lrcrrrrr}
\hline \hline & $\begin{array}{r}\text { AD } \\
\text { (Th) } \\
\text { dex }\end{array}$ & $\begin{array}{r}\text { Shift } \\
\mathrm{km} \mathrm{s}^{-1}\end{array}$ & $\begin{array}{r}V_{\text {br }} \\
\mathrm{km} \mathrm{s}^{-1}\end{array}$ & $\begin{array}{r}A(\mathrm{Th}) \\
\mathrm{dex}\end{array}$ & $\begin{array}{r}\text { SHIFT } \\
\mathrm{km} \mathrm{s}^{-1}\end{array}$ & $\begin{array}{r}V_{\text {br }} \\
\mathrm{km} \mathrm{s}^{-1}\end{array}$ & $\begin{array}{r}3 \mathrm{D}-1 \mathrm{D}_{\text {LHD }} \\
\text { dex }\end{array}$ \\
\hline Int. (Neckel) & 0.088 & -0.05 & 1.49 & 0.160 & -0.53 & 2.77 & -0.072 \\
Int. (Delbouille) & 0.080 & +0.01 & 1.86 & 0.168 & -0.47 & 2.99 & -0.088 \\
Flux (Neckel) & 0.056 & -0.39 & 1.68 & 0.168 & -0.47 & 4.11 & -0.112 \\
Flux (Kurucz) & 0.067 & -0.38 & 2.82 & 0.175 & -0.46 & 4.71 & -0.108 \\
\hline
\end{tabular}

Note: (2) - (4) results of the fitting: $A(\mathrm{Th})$, shift, and broadening using a 3D grid; (5)-(7) same for $1 \mathrm{D}_{\mathrm{LHD}}$ grid; (8) gives the difference in abundance $A(\mathrm{Th})_{3 \mathrm{D}}-A(\mathrm{Th})_{1 \mathrm{D}_{\mathrm{LHD}}}$.

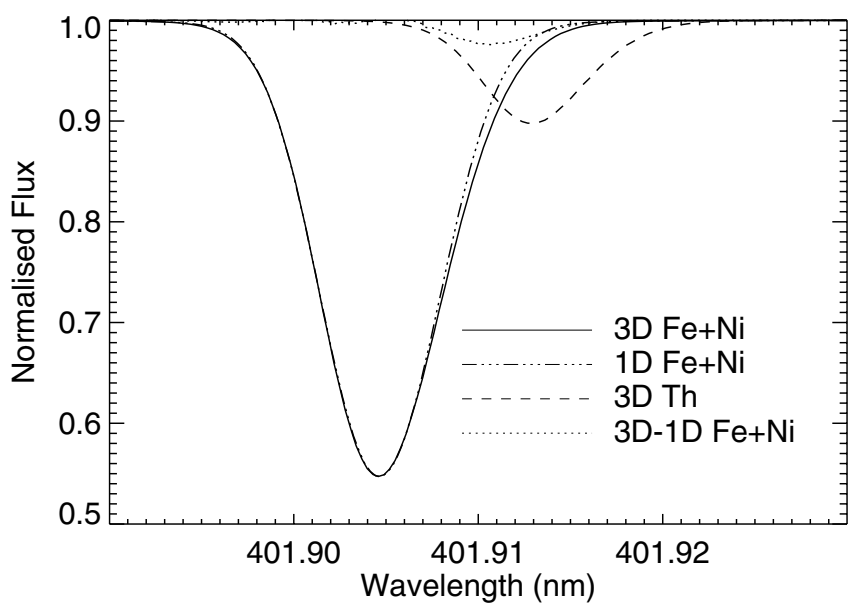

Fig. 2. The Fe I+Ni I blend in the 3D simulation (solid line) is compared to the $1 \mathrm{D}_{\mathrm{LHD}}$ profile (dash-dotted line), scaled in order to have the same central residual intensity as the $3 \mathrm{D}$ profile. The difference of the two spectra is shown as well as a Th $3 \mathrm{D}$ line profile.

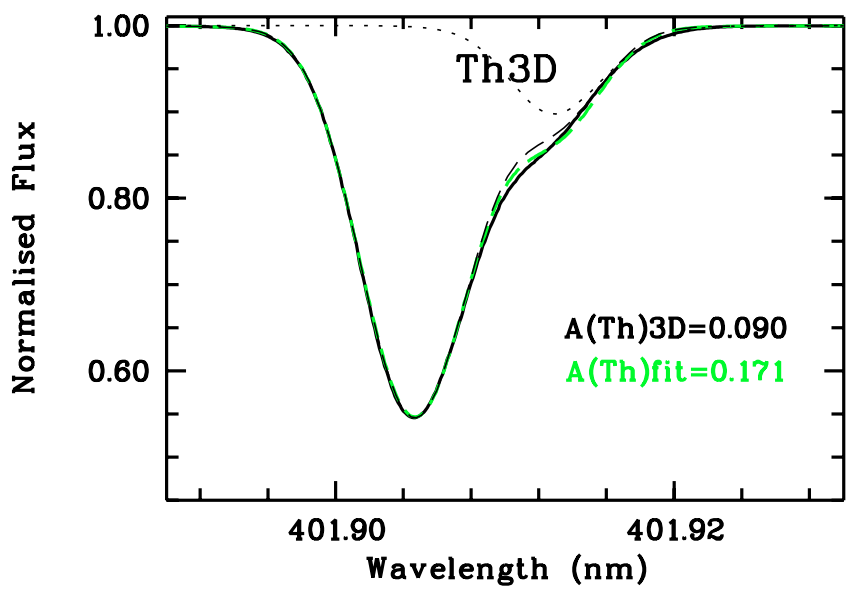

Fig. 3. The 3D profile of $(\mathrm{Fe} I+\mathrm{Ni} I)+(\mathrm{Th}$ II $+\mathrm{Co} I+\mathrm{V}$ II $)$ blend in solid black is over-imposed on the $1 \mathrm{D}_{\mathrm{LHD}}$ fit (green/grey dashed line). In the plot is also over-imposed (black dashed line) the $1 \mathrm{D}_{\mathrm{LHD}}$ with the same abundance as the $3 \mathrm{D}$ profile as well as the $3 \mathrm{D}$ Th II $+\mathrm{Co}$ I + V II profile (dotted line).

difference between the $3 \mathrm{D}$ and the $1 \mathrm{D}_{\text {LHD }}$ profiles is shown in the dotted line of the figure. The 3D thorium profile is also shown as a dashed line and it makes clear that the line asymmetry does indeed contribute to the measured $E W$. The $E W$ of this difference is $0.134 \mathrm{pm}$, which translates into a difference in abundance of +0.13 dex for flux. For intensity, $\Delta(E W)=0.088 \mathrm{pm}$ implies $\triangle A(\mathrm{Th})=+0.10$ dex in Th abundance.
On a level of slightly higher sophistication with respect to the above naive argument, we fitted the $3 \mathrm{D}$ synthetic profile with a grid of $1 D_{\text {LHD }}$ synthetic spectra of differing Th abundance. We scaled the $1 \mathrm{D}_{\mathrm{LHD}} \mathrm{Fe}+\mathrm{Ni}$ blend in order to induce it to have the same central residual intensity as the 3D profile. One of the results of the fitting is shown in Fig. 3: the 3D profile (black solid line) with $A(\mathrm{Th})=0.09$ when fitted with a $1 \mathrm{D}_{\text {LHD }}$ grid (dashed green/grey line) gives a higher abundance $A(\mathrm{Th})=0.171$. The use of a $\langle 3 \mathrm{D}\rangle$ grid leads to an even higher thorium abundance: $A(\mathrm{Th})=0.227$. In the plot the $1 \mathrm{D}_{\mathrm{LHD}}$ flux profile, with the same abundance as the $3 \mathrm{D}$ synthetic spectrum, is shown, as well as the $3 \mathrm{D}$ thorium profile. For the disc-centre intensity, with the same procedure as used for flux, the fitting of a 3D profile assuming a thorium abundance of 0.09 with a $1 \mathrm{D}_{\text {LHD }}$ grid implies $A(\mathrm{Th})=0.110$, while with a $\langle 3 \mathrm{D}\rangle$ grid $A(\mathrm{Th})=0.155$. The difference of the result of the fit when using $\langle 3 \mathrm{D}\rangle$ or $1 \mathrm{D}_{\mathrm{LHD}}$ grids is due to the superposition of the effect of the line asymmetry and the "intrinsic" $3 \mathrm{D}$ corrections $\left(A(\mathrm{Th})_{3 \mathrm{D}}-A(\mathrm{Th})_{1 \mathrm{D}}\right)$.

The Fe I+Ni I line asymmetry and the 3D corrections act in opposite directions: the line asymmetry produces a higher $A(\mathrm{Th})$ from $1 \mathrm{D}$ analysis, while the $3 \mathrm{D}$ correction is positive, thus $A$ (Th) from a $1 \mathrm{D}$ analysis is smaller. However, the $3 \mathrm{D}$ correction in intensity is between 3 and 4 times larger than for flux (see Table 4).

In Fig. 4 we show the results of fitting the whole spectral region both with $3 \mathrm{D}$ (left hand side) and 1D $\mathrm{D}_{\mathrm{LHD}}$ (right hand side) profiles. The synthetic profiles have been built by adding 5 components: a MnI+Fe I blend centred at $401.89 \mathrm{~nm}$, the Fe I+Ni I blend, the Th II+Co I+V II blend, an artificial Fe II line at 401.9206, initially suggested by François et al. (1993) and adopted also by del Peloso et al. (2005a), and the two Co I lines at 401.93. Of these components the $\mathrm{Th}$ II $+\mathrm{Co}$ I $+\mathrm{V}$ II blend was computed for three different Th abundances, all the other components were instead computed for a single abundance and were scaled by the fitting procedure in order to best reproduce the observed spectrum.

The results of the fitting are summarised in Table 5. From the table one sees that the total 3D correction, taking into account both line asymmetry and "intrinsic" 3D correction is from -0.07 to -0.09 dex for the intensity spectra and -0.11 dex for the flux spectra. This difference is expected, given that the "intrinsic" 3D correction, as given in Table 4 , is about +0.02 dex for intensity and about +0.005 dex for flux.

It is quite interesting to note that in the case of Th the line asymmetry implies a total $3 \mathrm{D}$ effect which is not only much larger, but also in a different direction, from what would have been implied by the "intrinsic" 3D correction. To our knowledge this is the second time such an effect has been highlighted. These "total" 3D corrections are only slightly larger than what was estimated above from the fit of the 3D Fe I+Ni I blend plus Th II + Co I + V II blend, with $1 \mathrm{D}_{\text {LHD }}$ profiles. This suggests that the use of the simpler procedure is still capable of providing the 


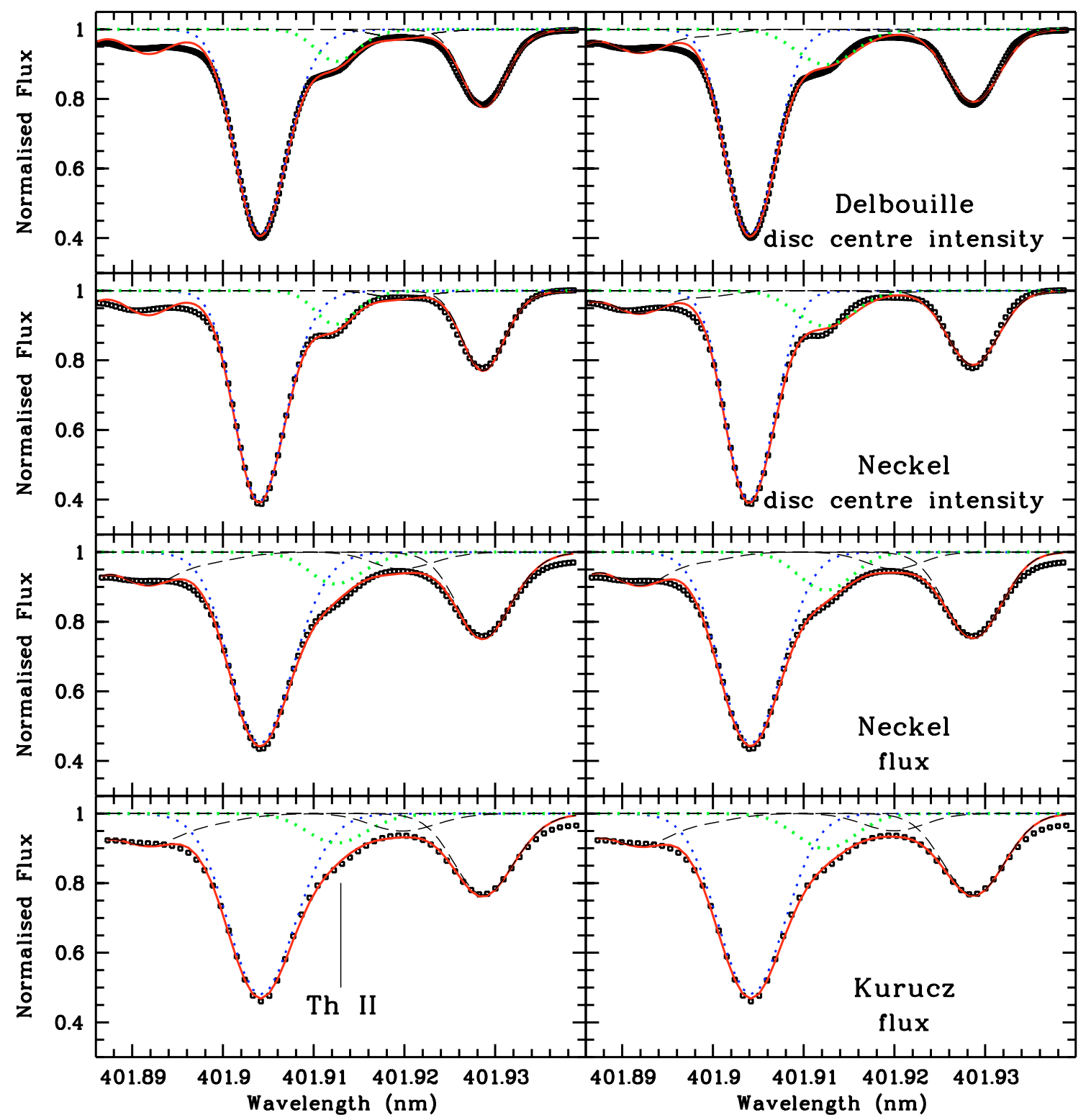

Fig. 4. Fits of the synthetic spectra to the observed solar spectra. On the left hand side, the synthetic spectra have been computed from the $\mathrm{CO}^{5} \mathrm{BOLD}$ model, on the right hand side from the corresponding $1 \mathrm{D}_{\mathrm{LHD}}$ model. On either side, from top to bottom, the spectra are: the Delbouille disc centre intensity, the Neckel disc centre intensity, the Neckel flux and the Kurucz flux. On each panel the sub-components used to synthesise the feature are shown individually: the light dotted line is the Th II+Co I+V II blend, the darker dotted line is the Ni I+Fe I blend, the black dashed lines are, from blue to red, the Mn I+Fe I blend the artificial Fe II line and the Co I $401.93 \mathrm{~nm}$ lines.

correct order of magnitude for the "total" 3D correction. Finally we point out that, as can be seen from the left hand side of Fig. 4, it is obvious that the $1 \mathrm{D}_{\mathrm{LHD}}$ profiles are unable to provide the correct shape for the Th feature. The shape is reproduced in a much more satisfactory way by the $3 \mathrm{D}$ profiles.

We conclude this section by investigating the iron line at $401.9 \mathrm{~nm}$, which is the stronger component of the Fe I+Ni I blend. The 3D synthetic line shows a strong asymmetry; the difference 3D-1D is very pronounced on the red wing if we make an appropriate shift and broadening to the 1D profile so as to over-impose the blue wings of the 3D and 1D synthetic spectra. While the flux of the 3D synthetic spectrum is shifted to the blue by $0.1 \mathrm{~km} \mathrm{~s}^{-1}$, the intensity spectra, corresponding to different inclination angles, show a shift ranging from $-0.5 \mathrm{~km} \mathrm{~s}^{-1}$ to $+0.15 \mathrm{~km} \mathrm{~s}^{-1}$.

\section{The 3D effects for other models.}

\subsection{Hafnium}

Beyond the solar model as such we investigated the hydrodynamical effects on some other 3D models of dwarf and subgiant stars (see Table 2). We restricted our investigation to solar or -1.0 metallicity, because for lower metallicity these hafnium lines are no longer measurable in dwarfs. Even the detection at -1.0 metallicity is only possible for the strongest of the hafnium lines, but the two metallicities can be used for interpolation to intermediate metallicity. We find that 3D and 1D abundances are generally very similar, making the 3D corrections insignificant for all models and all lines we consider.

$\mathrm{Hf}$ II is the dominant species of hafnium in the photosphere of these models. Both the $5900 \mathrm{~K}$ models predict that at least 
Table 6. Non-solar $\mathrm{CO}^{5} \mathrm{BOLD}$ models considered in this work.

\begin{tabular}{|c|c|c|c|c|c|c|c|c|}
\hline $\begin{array}{r}\text { Wavelength } \\
\mathrm{nm} \\
(1)\end{array}$ & $\begin{array}{l}E W \\
\text { pm } \\
(2)\end{array}$ & $\begin{array}{l}3 \mathrm{D} \\
\text { (3) }\end{array}$ & $\begin{array}{r}A(\mathrm{Hf}) \\
\langle 3 \mathrm{D}\rangle \\
(4)\end{array}$ & $\begin{array}{r}1 \mathrm{D}_{\text {LHD }} \\
(5)\end{array}$ & $\begin{array}{r}3 \mathrm{D}-\langle 3 \mathrm{D}\rangle \\
(6)\end{array}$ & $\begin{array}{r}3 \mathrm{D}-1 \mathrm{D}_{\mathrm{LHD}} \\
\text { (7) }\end{array}$ & $\begin{array}{r}\sigma_{\langle E W\rangle} \\
\% \\
(8)\end{array}$ & $\begin{array}{l}\sigma_{\mathrm{A}} \\
\text { dex } \\
(9)\end{array}$ \\
\hline \multicolumn{9}{|l|}{$5060 / 4.5 / 0.0$} \\
\hline 339.0 & 0.718 & 0.870 & 0.842 & 0.829 & 0.028 & 0.041 & 0.05 & 0.0002 \\
\hline 356.2 & 1.550 & 0.872 & 0.809 & 0.796 & 0.062 & 0.076 & 0.09 & 0.0003 \\
\hline 391.8 & 0.405 & 0.870 & 0.854 & 0.841 & 0.016 & 0.029 & 0.07 & 0.0003 \\
\hline 409.3 & 0.410 & 0.870 & 0.855 & 0.841 & 0.016 & 0.029 & 0.07 & 0.0003 \\
\hline \multicolumn{9}{|l|}{$5060 / 4.5 /-1.0$} \\
\hline 339.0 & 0.151 & 0.870 & 0.866 & 0.852 & 0.004 & 0.018 & 0.11 & 0.0005 \\
\hline 356.2 & 0.395 & 0.870 & 0.864 & 0.852 & 0.007 & 0.018 & 0.12 & 0.0005 \\
\hline 391.8 & 0.080 & 0.870 & 0.870 & 0.855 & 0.001 & 0.015 & 0.11 & 0.0005 \\
\hline 409.3 & 0.081 & 0.868 & 0.867 & 0.853 & 0.001 & 0.015 & 0.11 & 0.0005 \\
\hline \multicolumn{9}{|l|}{$5870 / 4.5 / 0.0$} \\
\hline 339.0 & 0.551 & 0.870 & 0.862 & 0.858 & 0.008 & 0.011 & 0.20 & 0.0008 \\
\hline 356.2 & 1.150 & 0.870 & 0.857 & 0.849 & 0.012 & 0.020 & 0.22 & 0.0010 \\
\hline 391.8 & 0.330 & 0.871 & 0.867 & 0.869 & 0.004 & 0.002 & 0.18 & 0.0008 \\
\hline 409.3 & 0.336 & 0.870 & 0.867 & 0.867 & 0.003 & 0.003 & 0.18 & 0.0008 \\
\hline \multicolumn{9}{|l|}{$5920 / 4.5 /-1.0$} \\
\hline 3390 & 0.095 & 0.871 & 0.872 & 0.868 & -0.001 & 0.003 & 0.57 & 0.0025 \\
\hline 3562. & 0.230 & 0.870 & 0.880 & 0.881 & -0.010 & -0.011 & 0.67 & 0.0029 \\
\hline 3918. & 0.053 & 0.869 & 0.872 & 0.873 & -0.003 & -0.004 & 0.55 & 0.0024 \\
\hline 4093. & 0.054 & 0.866 & 0.869 & 0.870 & -0.003 & -0.004 & 0.56 & 0.0026 \\
\hline \multicolumn{9}{|l|}{$6230 / 4.5 / 0.0$} \\
\hline 339.0 & 0.444 & 0.870 & 0.865 & 0.859 & 0.005 & 0.012 & 0.22 & 0.0009 \\
\hline 356.2 & 0.916 & 0.870 & 0.865 & 0.855 & 0.004 & 0.015 & 0.25 & 0.0011 \\
\hline 391.8 & 0.275 & 0.870 & 0.866 & 0.870 & 0.004 & -0.001 & 0.19 & 0.0008 \\
\hline 409.3 & 0.281 & 0.870 & 0.866 & 0.869 & 0.003 & 0.001 & 0.20 & 0.0009 \\
\hline \multicolumn{9}{|l|}{$6240 / 4.5 /-1.0$} \\
\hline 339.0 & 0.073 & 0.869 & 0.876 & 0.870 & -0.007 & -0.001 & 0.19 & 0.0008 \\
\hline 356.2 & 0.173 & 0.871 & 0.889 & 0.889 & -0.018 & -0.018 & 0.20 & 0.0009 \\
\hline 391.8 & 0.042 & 0.866 & 0.874 & 0.876 & -0.008 & -0.010 & 0.17 & 0.0007 \\
\hline 409.3 & 0.044 & 0.875 & 0.884 & 0.885 & -0.009 & -0.010 & 0.17 & 0.0007 \\
\hline \multicolumn{9}{|l|}{$6460 / 4.5 / 0.0$} \\
\hline 339.0 & 0.374 & 0.871 & 0.869 & 0.864 & 0.002 & 0.006 & 0.23 & 0.0010 \\
\hline 356.2 & 0.764 & 0.870 & 0.871 & 0.863 & 0.000 & 0.007 & 0.26 & 0.0011 \\
\hline 391.8 & 0.239 & 0.869 & 0.868 & 0.877 & 0.002 & -0.008 & 0.20 & 0.0009 \\
\hline 409.3 & 0.245 & 0.871 & 0.869 & 0.877 & 0.002 & -0.006 & 0.20 & 0.0009 \\
\hline \multicolumn{9}{|l|}{$6460 / 4.5 /-1.0$} \\
\hline 339.0 & 0.059 & 0.867 & 0.883 & 0.875 & -0.016 & -0.007 & 0.25 & 0.0011 \\
\hline 356.2 & 0.137 & 0.868 & 0.897 & 0.897 & -0.029 & -0.029 & 0.27 & 0.0012 \\
\hline 391.8 & 0.035 & 0.865 & 0.881 & 0.882 & -0.016 & -0.017 & 0.21 & 0.0009 \\
\hline 409.3 & 0.036 & 0.866 & 0.883 & 0.883 & -0.016 & -0.017 & 0.21 & 0.0009 \\
\hline \multicolumn{9}{|l|}{$5500 / 3.5 / 0.0$} \\
\hline 339.0 & 1.500 & 0.868 & 0.855 & 0.850 & 0.014 & 0.019 & 0.23 & 0.0010 \\
\hline 356.2 & 2.780 & 0.871 & 0.845 & 0.830 & 0.026 & 0.041 & 0.29 & 0.0012 \\
\hline 391.8 & 0.987 & 0.870 & 0.865 & 0.867 & 0.005 & 0.003 & 0.21 & 0.0009 \\
\hline \multirow{2}{*}{\multicolumn{9}{|c|}{$5500 / 3.5 /-1.0$}} \\
\hline & & & & & & & & \\
\hline 339.0 & 3.160 & 0.870 & 0.870 & 0.863 & 0.001 & 0.007 & 0.29 & 0.0013 \\
\hline 356.2 & 0.758 & 0.870 & 0.874 & 0.862 & -0.003 & 0.008 & 0.34 & 0.0015 \\
\hline 391.8 & 0.182 & 0.871 & 0.874 & 0.872 & -0.003 & -0.001 & 0.28 & 0.0012 \\
\hline 409.3 & 0.186 & 0.871 & 0.875 & 0.870 & -0.004 & 0.001 & 0.29 & 0.0013 \\
\hline
\end{tabular}

Note: The atmospheric parameters are given as $T_{\mathrm{eff}} / \log g /[\mathrm{M} / \mathrm{H}]$. Columns $(3)-(5)$ are the $A(\mathrm{Hf})$, according to $\mathrm{CO}^{5} \mathrm{BOLD} 3 \mathrm{D},\langle 3 \mathrm{D}\rangle$ and $1 \mathrm{D}_{\mathrm{LHD}}$ model; Cols. (6)-(7) are 3D corrections, 3D- $\langle 3 \mathrm{D}\rangle$ and 3D-1D $\mathrm{D}_{\mathrm{LHD}}$ respectively; Col. (8) statistical uncertainty of the theoretical $E W$; Col. (9) corresponding uncertainty in abundance.

$98 \%$ of the Hf is ionised. The hotter models foresee an even lower fraction of $\mathrm{Hf}$ I. The coolest models at $5000 \mathrm{~K}$ predict that at least $75 \%$ of the Hf is ionised. The results are similar when considering the $\langle 3 \mathrm{D}\rangle$ or the $1 \mathrm{D}_{\mathrm{LHD}}$ temperature profile for the atmosphere.

If we compare the horizontal average temperature profile of the $3 \mathrm{D}$ model to the $1 \mathrm{D}_{\mathrm{LHD}}$ model we can see that they are very similar. There is no pronounced cooling of the $3 \mathrm{D}$ atmospheres relative to $1 D_{L H D}$ in radiative equilibrium for the same atmospheric parameters which is known to be prominent in more metal-poor models.

\subsection{Thorium}

As for hafnium and as in the solar case, the "intrinsic" 3D corrections are small for the other models. The hydrodynamical effects are instead due to the line asymmetry, like in 
the Sun. When we fit, as we do for the Sun, a 3D flux profile, with meteoritic or scaled meteoritic $A(\mathrm{Th})$ for the metal-poor models, with a $1 \mathrm{D}_{\mathrm{LHD}}$ grid we obtain for $A$ (Th) a value higher by $0.08-0.12$ dex considering the models: $5500 \mathrm{~K} / 3.50 / 0.0,5500 \mathrm{~K} / 3.50 /-1.05900 \mathrm{~K} / 4.00 / 0.0$, $5900 \mathrm{~K} / 4.00 /-1.0,5900 \mathrm{~K} / 4.50 / 0.0$, and $5900 \mathrm{~K} / 4.50 /-1.0$ (see Table 2). One has to keep in mind that such differences translate into systematic age differences of almost $5 \mathrm{Gyr}$ in the context of nucleocosmochronology.

\section{Conclusions}

Our determination of the photospheric hafnium abundance, $A(\mathrm{Hf})=0.87 \pm 0.04$, derived from a $3 \mathrm{D}$ hydrodynamical $\mathrm{CO}^{5} \mathrm{BOLD}$ solar model, is very close to the abundance determination of Lawler et al. (2007) obtained using the HolwegerMüller solar model. The $E W \mathrm{~s}$ of the hafnium lines are not very sensitive to the hydrodynamical effects. A difference of the same amount of the 3D correction can be found if we compare the $A(\mathrm{Hf})$ obtained using two different $1 \mathrm{D}$ models, such as ATLAS and Holweger-Müller model or $1 \mathrm{D}_{\mathrm{LHD}}$ and the Holweger-Müller model. These difference are related to different opacities and physics used to compute the models. Synthetic profiles of hafnium lines computed from 3D models show a pronounced asymmetry, but this effect is not relevant for abundance determination as long as only the $E W$ is considered.

For thorium, as for hafnium, $3 \mathrm{D}$ effects on the $E W \mathrm{~s}$ are less than or comparable to the uncertainties related to the $E W$ measurement, so that they are negligible when determining $A(\mathrm{Th})$. However, for the solar photospheric thorium determination the asymmetry of the strong Fe I+Ni I line blending the $401.9 \mathrm{~nm}$ Th II weak line implies a non-negligible effect on the derived Th abundance and is in fact the dominant source of the "total" $3 \mathrm{D}$ effect. The asymmetry is a hydrodynamical effect, and we find a difference in the $A(\mathrm{Th})$ determination of about $-0.1 \mathrm{dex}$ when considering this asymmetry. Our results for the Sun and for other 3D dwarfs and sub-giants models imply that Th cannot be reliably measured without making use of hydrodynamical simulation if the Th line is weak and blended. A reappraisal of existing Th measurements in the light of hydrodynamical simulations is warranted for dwarfs and sub-giants.
Acknowledgements. The authors L.S., H.-G.L., P.B, and N.T.B. acknowledge financial support from EU contract MEXT-CT-2004-014265 (CIFIST).

\section{References}

Andersen, T., Petersen, P., \& Hauge, O. 1976, Sol. Phys., 49, 211 Anders, E., \& Grevesse, N. 1989, Geochim. Cosmochim. Acta, 53, 197 Arlandini, C., Käppeler, F., Wisshak, K., et al. 1999, ApJ, 525, 886 Caffau, E., \& Ludwig, H.-G. 2007, A\&A, 467, L11

Caffau, E., Faraggiana, R., Bonifacio, P., Ludwig, H.-G., \& Steffen, M. 2007a, A\&A, 470, 699

Caffau, E., Steffen, M., Sbordone, L., Ludwig, H.-G., \& Bonifacio, P. 2007b, A\&A, 473, L9

Cayrel, R., Hill, V., Beers, T. C., et al. 2001, Nature, 409, 691

Cayrel, R., Steffen, M., Chand, H., et al. 2007, A\&A, 473, L37

Cowan, J. J., Pfeiffer, B., Kratz, K.-L., et al. 1999, ApJ, 521, 194

del Peloso, E. F., da Silva, L., \& Porto de Mello, G. F. 2005, A\&A, 434, 275

Delbouille, L., Roland, G., \& Neven, L. 1973, Photometric Atlas of the Solar Spectrum from $\lambda \lambda 3000$ to $\lambda \lambda 10000$ (Liège: Univ. Liège, Institut d'Astrophysique)

François, P., Spite, M., \& Spite, F. 1993, A\&A, 274, 821

Frebel, A., Christlieb, N., Norris, J. E., et al. 2007, ApJ, 660, L117

Freytag, B., Steffen, M., \& Dorch, B. 2002, Astron. Nachr., 323, 213

Grevesse, N., Noels, A., \& Sauval, A. J. 1996, Cosmic Abundances, 99, 117

Grevesse, N., \& Sauval, A. J. 1998, Space Sci. Rev., 85, 161

Hill, V., Plez, B., Cayrel, R., et al. 2002, A\&A, 387, 560

Holweger, H. 1967, Z. Astrophys., 65, 365

Holweger, H. 1980, The Observatory, 100, 155

Holweger, H., \& Mueller, E. A. 1974, Sol. Phys., 39, 19

Ivans, I. I., Simmerer, J., Sneden, C., et al. 2006, ApJ, 645, 613

Kratz, K.-L., Farouqi, K., Pfeiffer, B., et al.2007, ApJ, 662, 39

Kurucz, R. L. 2005, MSAIS, 8, 189

Lawler, J. E., Whaling, W., \& Grevesse, N. 1990, Nature, 346, 635

Lawler, J. E., Hartog, E. A. D., Labby, Z. E., et al. 2007, ApJS, 169, 120

Lodders, K. 2003, ApJ, 591, 1220

Ludwig, H.-G., Caffau, E., Steffen, M., \& Bonifacio, P. 2008a, in preparation

Ludwig, H.-G., Steffen, M., Freytag, B., et al. 2008b, in preparation

Neckel, H., \& Labs, D. 1984, Sol. Phys., 90, 205

Rocholl, A., \& Jochum, K. P. 1993, Earth Planet. Sci. Lett., 117, 265

Russell, H. N. 1929, ApJ, 70, 11

Sneden, C., Johnson, J., Kraft, R. P., et al. 2000, ApJ, 536, L85

Svensson, F., \& Ludwig, H.-G. 2005, 13th Cambridge Workshop on Cool Stars, Stellar Systems and the Sun, 560, 979

Wedemeyer, S., Freytag, B., Steffen, M., Ludwig, H.-G., \& Holweger, H. 2004, A\&A, 414, 1121 